\title{
The Close Environment of Z CMa Binary System
}

\author{
Paulo J. V. Garcia \\ Centro de Astrofísica da Universidade do Porto, Rua das Estrelas, \\ 4150-762 Porto, Portugal; Isaac Newton Group of Telescopes, Spain; \\ CRAL/Observatoire de Lyon, CNRS UMR 5574, France.
}

\begin{abstract}
We present echelle spectroastrometric observations of the $\mathrm{Z}$ CMa system in the $\mathrm{H} \alpha$ region showing its feasibility and underlining the astrophysical importance of the information stored in the spatial width of the spectra.

The $\mathrm{H} \alpha$ profile dating from 12/1991 presents a "bullet" of blueshifted emission superimposed on the broad absorption component. We interpret this material as an unresolved bullet of gas at the base of the jet. We show that the jet emission fills in the absorption component of the $\mathrm{H} \alpha$ profile and has a wide range of velocities.
\end{abstract}

\section{Introduction}

$\mathrm{Z} \mathrm{CMa}$ is a pre-main-sequence binary with a projected separation of $0.1^{\prime \prime}$, i.e., $\sim 100$ AU (Koresko et al. 1991). The interest in Z CMa lies in its components: an FU Ori star and an embedded Herbig Ae/Be star (Welty et al. 1989; Whitney et al. 1993). Furthermore it drives a powerful jet extending up to $2 \mathrm{pc}$ away (Poetzel et al. 1985). The source of the outflow is the embedded Herbig Ae/Be star (Garcia et al. 1999).

The spectrum of the system is quite complex and consists of the superposition of the FU Ori and Herbig Ae/Be components, the latter dominating the emission lines (Whitney et al., Garcia et al.). Furthermore it is variable with a peak of activity in the 1987 outburst (Hessman et al. 1991). The source of variability is essentially the Herbig Ae/Be star (which drives the outflow).

Speckle polarimetry of the system found that both components have strong polarization in the $\mathrm{K}$ band, and this was interpreted that each has a more pole-on than edge-on configuration of the jet and disk axis (Fischer et al. 1998).

In the following section we introduce the concept of spectroastrometry and apply it to echelle spectra of Z CMa.

\section{Spectroastrometry}

The concept of spectroastrometry dates back to the early 80 s (see Bailey, 1998 for references to the early literature). The idea is quite straightforward and we will introduce it for the familiar case of long-slit spectroscopy. Consider a long-slit spectrum of a single star. One of the CCD directions is the dispersion direction $(y)$ and the other is the spatial direction $(x)$. A normal step in the spectral extraction is to trace the spectra. We are interested in a very precise 
measurement of the spectra center at each $y$. In order to obtain it a Gaussian is fit to the spatial distribution thus measuring a centroid $x_{c}, \sigma$, and amplitude $A^{1}$. The previous quantities are actually functions of $y: A(y), x_{c}(y)$ and $\sigma(y)$. The final precision in the centroid is roughly $\delta\left(x_{c}\right) \sim \Delta / S N R$, where $\Delta$ is the pixel size and $S N R$ the signal-to-noise ratio in the central pixel. Supposing a pixel size of $0.27^{\prime \prime}$ and a $S N R=100$, the precision $\delta\left(x_{c}\right)$ is thus 3 milliarcsec. In practice the spectrograph presents some distortion that must be corrected for generally by taking another spectrum with the slit rotated by $180^{\circ}$. The atmospheric differential refraction must also be corrected - for high resolution spectra it can be removed by straight line fitting. Finally we are left with the residuals where the astrometric information is stored versus wavelength. Another piece of information, often ignored, is the behaviour of $\sigma$ with wavelength which can give us crucial information on extended structure.

The secret of spectroastrometry lies in its differential nature, we measure relative shifts $/ \sigma$ in emission lines with respect to the continuum. The main difficulty lies in the interpretation of the observations which is greatly simplified for simple objects like binaries. This technique has been used to search for premain-sequence binaries by Bailey 1998, and Garcia et al. 1999 showed that the combination of resolved speckle photometry with spectroastrometry allows the recovery of resolved spectroscopy for binaries as close as $0.1^{\prime \prime}$.

\section{Echelle Spectroastrometry of Z CMa}

In Figure 1 we present an application of spectroastrometry to an echelle spectrum of $\mathrm{Z} \mathrm{CMa}$ in the $\mathrm{H} \alpha$ order. The procedure followed was the same as presented above. The spectrum presents an emission peak and broad P Cyg like profile quite similar to the one presented in Reipurth et al. (1996), with a further emission component which we estimate as located at $\gtrsim 100 \mathrm{~km} / \mathrm{s}$ and will call "bullet". This "bullet" component is still present in another spectrum taken 25 hours later and thus originates in a zone with size $z 13 R_{\odot}$. We note that the observed spectra, and in particular the $\mathrm{H} \alpha$ profile, are due to the superposition of the profiles of both components plus the jet emission.

The centroid as a function of wavelength shifts towards the north (the projected direction of the Herbig $\mathrm{Ae} / \mathrm{Be}$ star) at the position of the $\mathrm{H} \alpha$ profile. This is expected due to the strong contrast between the $\mathrm{H} \alpha$ profiles of the FU Ori (absorption) and Herbig Ae/Be (emission) components. Bailey's $\mathrm{H} \alpha$ spectroastrometry of Z CMa for the NS direction shows instead a double peak structure. The difference is probably due to the absence of the "bullet" component in his 1997 spectra. This component originates in the jet source and will contribute to the centroid shift.

Important information is also present in the Gaussian $\sigma$ whose differential nature makes it sensitive to the relative sizes of the various emission regions. In the absorption zone of the $\mathrm{H} \alpha$ profile the $\sigma$ increases, suggesting that the zone contributing to it is extended. Garcia et al. argued that the system micro-jet (1" long, see Figure 2) originated in the 1987 outburst. The echelle data was

\footnotetext{
${ }^{1}$ It is crucial to fit a Gaussian or some similar function (Moffat) because this is the most precise method to measure centroids.
} 

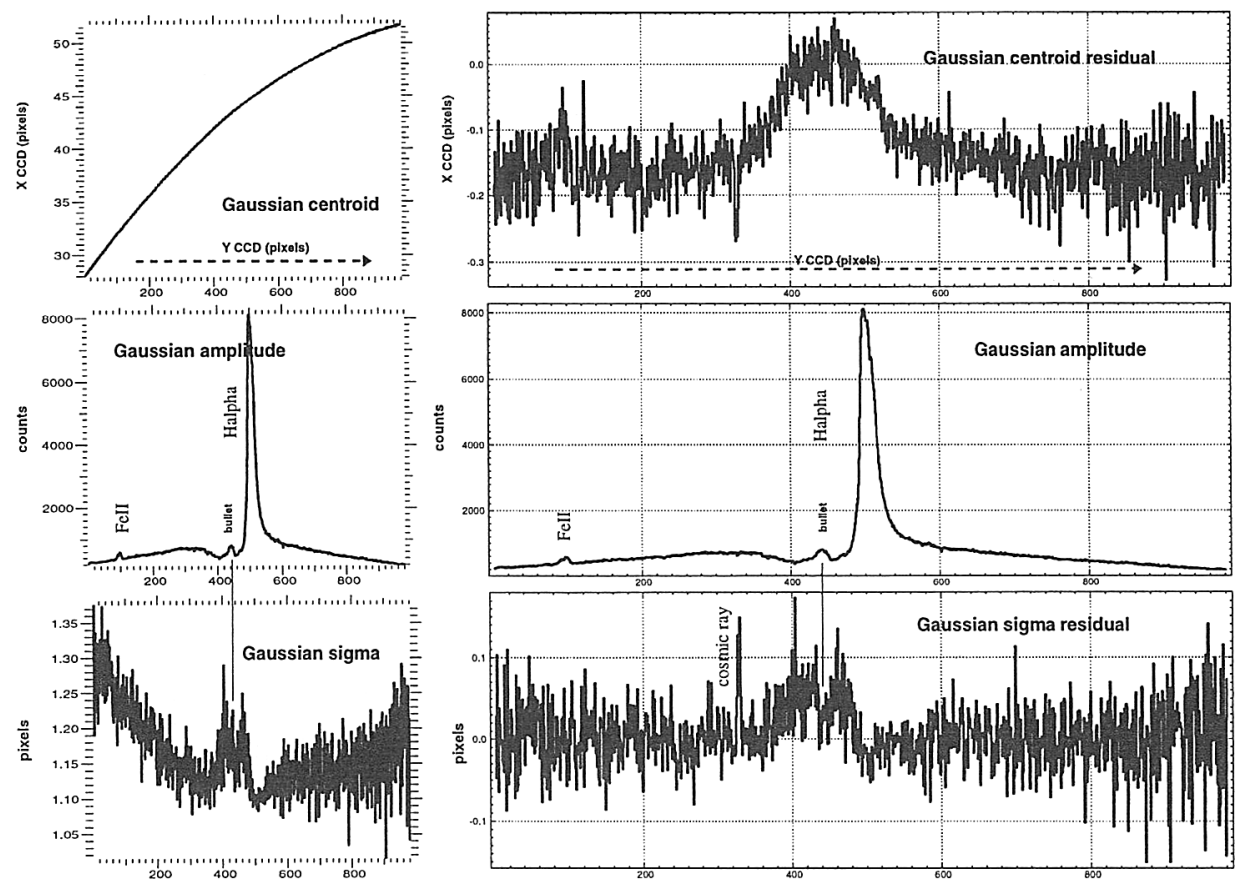

Figure 1. Spectroastrometry for an EMMI echelle order (R 28 000) containing the $\mathrm{H} \alpha$ line of $\mathrm{Z} \mathrm{CMa}$. The data were taken the 20/12/1991 (UT 03:34) at the NTT with the slit in the NS direction, pixel size $0.27^{\prime \prime}$. On the left we present the raw output of the Gaussian fits: $x_{c}, A$ and $\sigma$, on the right the calibrated residuals for $x_{c}$ and $\sigma$. For guidance we also plot the amplitude. To extract the residual $x_{c}$ and $\sigma$ we used as models of the echelle spatial distortion the two orders enclosing the $\mathrm{H} \alpha$ one, because all the available data had the slit in the NS direction. These residuals were obtained by subtracting from the $\mathrm{H} \alpha$ order centroid the average of the centroids for two orders enclosing it $\left(\operatorname{res}\left(x_{c}\right)=x_{c}^{H \alpha}-\left(x_{c}^{H \alpha-1}+x_{c}^{H \alpha+1}\right) / 2\right)$. The final precision ( $\left.\mathrm{rms}\right)$ in $\operatorname{res}\left(x_{c}\right)$ is 11 milliarcsec. The same procedure was applied to obtain the Gaussian $\sigma$ residuals: $\operatorname{res}(\sigma)=\sigma^{H \alpha}-\left(\sigma^{H \alpha-1}+\sigma^{H \alpha+1}\right) / 2$. The final precision ( $\mathrm{rms}$ ) in $\operatorname{res}(\sigma)$ is 17 milliarcsec. 

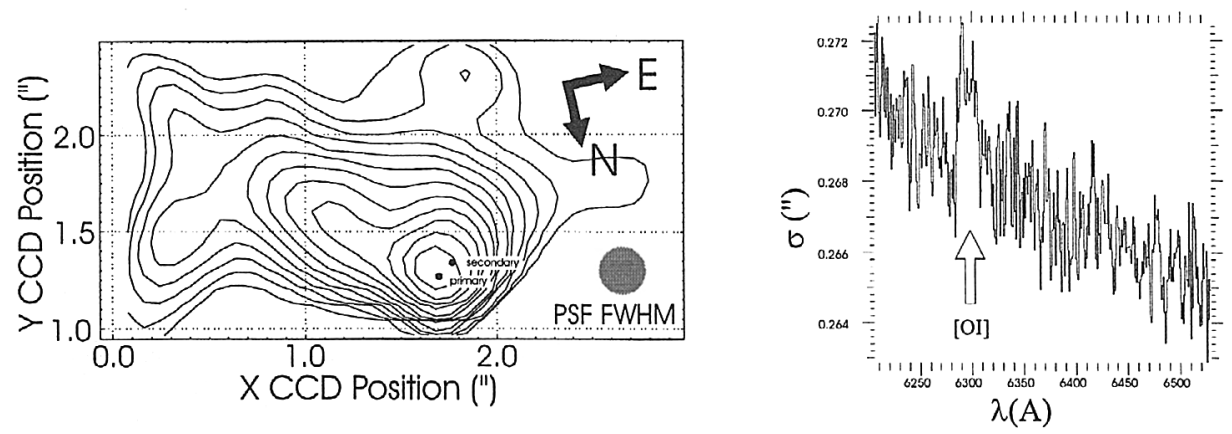

Figure 2. OASIS imaging and spectroastrometric data for $\mathrm{Z} \mathrm{CMa}$ (Garcia et al.) Left: the deconvolved jet image in the [OI]6300 emission line. Right: the evolution of the Gaussian $\sigma$ with wavelength. The overall $\sigma$ decreasing with wavelength is expected by seeing theory and better AO correction efficiency. The bump in $\sigma$ at the [OI]6300 position hints extended structure which is confirmed in the left image.

obtained 5 years later when the micro-jet should be $0.5^{\prime \prime}$ long. We are probably detecting it in the blue-shifted $\mathrm{H} \alpha$ zone. A similar effect is present in the $\sigma$ behaviour of the Gaussian fit to the Garcia et al. OASIS data (see Figure 2). The echelle data Gaussian $\sigma$ bump presents a small depression at the $\mathrm{H} \alpha$ bullet position. We interpret it as evidence that the bullet is situated very near the Herbig Ae/Be star and unresolved when compared to the micro-jet.

Acknowledgments. This research was partially supported by the Portuguese Fundação para a Ciência e Tecnologia through grant PRAXIS XXI / BPD / 20179 / 99. Partially based on archival observations made with ESO Telescopes at the La Silla Observatory under programme ID <48.7-0048>.

\section{References}

Bailey, J. 1998, MNRAS, 301, 161

Fischer, O., Stecklum, B., Leinert, Ch. 1998, A\&A, 334, 969

Garcia, P. J. V., Thiébaut, E., Bacon, R. 1999, A\&A, 346, 892.

Hartmann, L., Kenyon, S. J., Hewett, R. et al. 1989, ApJ, 338, 1001

Hessman, F. V., Eislöffel, J., Mundt, R. et al. 1991, ApJ, 370, 384

Koresko, C. D., Beckwith, S. V. W., Ghez, A. M. et al. 1991, AJ, 102, 6, 2073

Poetzel, R., Mundt, R., Ray, T. P. 1989, A\&A, 224, L13

Reipurth, B., Pedrosa, A., \& Lago, M. T. V. T. 1996, A\&AS, 120, 229

Whitney, B. A., Clayton, G. C., Shulte-Ladbeck, R. E., et al. 1993, ApJ 417, 687

Welty, A., Strom, S. E., Edwards, S., Kenyon, S. J., Hartmann, L. W. 1992, ApJ, 397, 260 

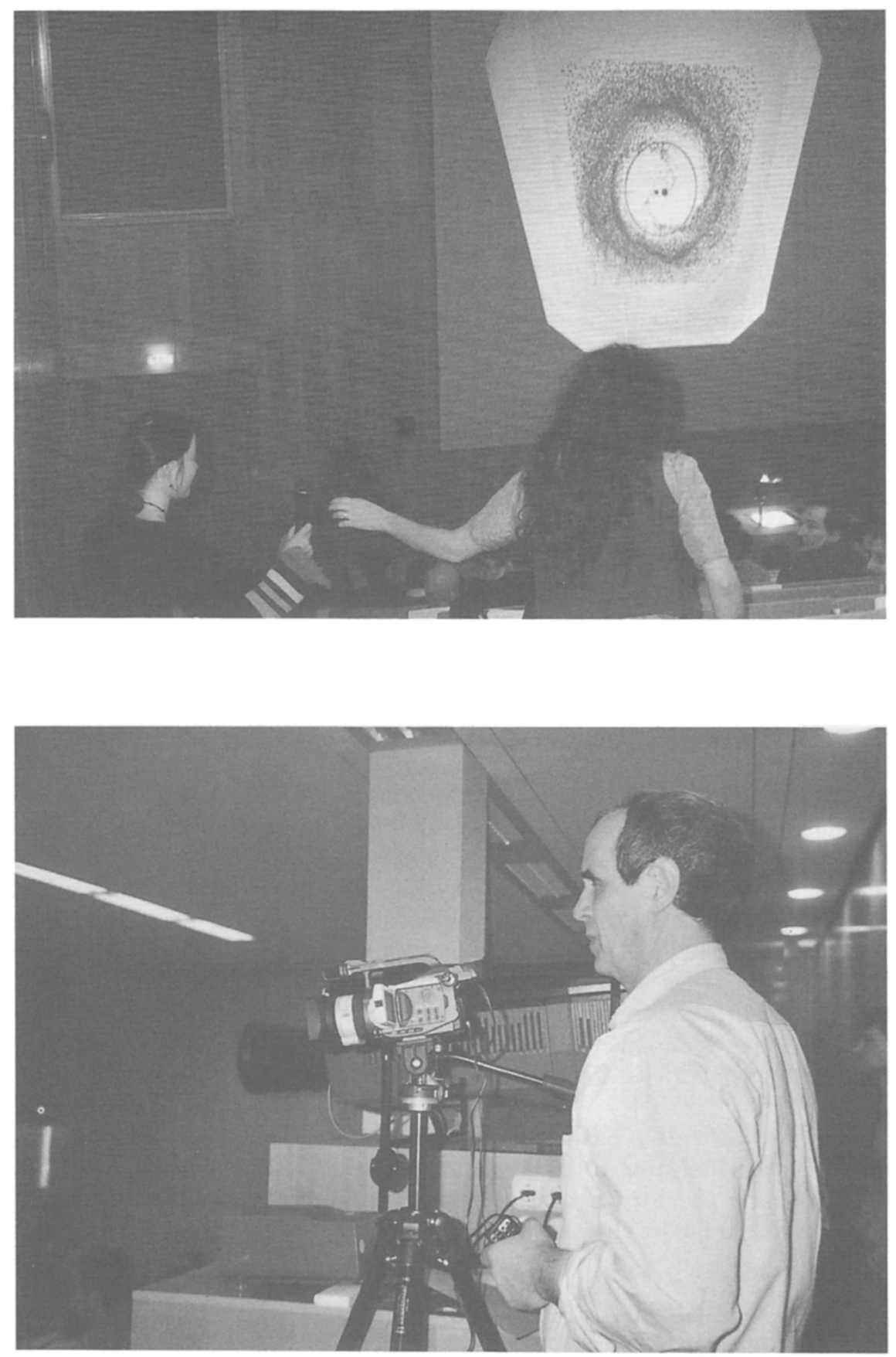

Cathie Clarke grabbing the microphone during a discussion on circumbinary disks (top)

Uli von Kusserow patiently taping the discussion on video (bottom) 